\title{
Flare Characteristics from X-ray Light Curves
}

\author{
M. Gryciuk ${ }^{1,2}$ (D) M. Siarkowski ${ }^{1} \cdot$ J. Sylwester ${ }^{1}$. \\ S. Gburek ${ }^{1}$ - P. Podgorski ${ }^{1}$ - A. Kepa ${ }^{1}$ B. Sylwester ${ }^{1}$. \\ T. Mrozek ${ }^{1,2}$
}

Received: 20 May 2016 / Accepted: 25 April 2017 / Published online: 6 June 2017

(C) The Author(s) 2017. This article is published with open access at Springerlink.com

\begin{abstract}
A new methodology is given to determine basic parameters of flares from their $\mathrm{X}$-ray light curves. Algorithms are developed from the analysis of small X-ray flares occurring during the deep solar minimum of 2009, between Solar Cycles 23 and 24, observed by the Polish Solar Photometer in X-rays (SphinX) on the Complex Orbital Observations Near-Earth of Activity of the Sun-Photon (CORONAS-Photon) spacecraft. One is a semiautomatic flare detection procedure that gives start, peak, and end times for single ("elementary") flare events under the assumption that the light curve is a simple convolution of a Gaussian and exponential decay functions. More complex flares with multiple peaks can generally be described by a sum of such elementary flares. Flare time profiles in the two energy ranges of SphinX $(1.16-1.51 \mathrm{keV}, 1.51-15 \mathrm{keV})$ are used to derive temperature and emission measure as a function of time during each flare. The result is a comprehensive catalogue - the SphinX Flare Catalogue - which contains 1600 flares or flare-like events and is made available for general use. The methods described here can be applied to observations made by Geosynchronous Operational Environmental Satellites (GOES), the Reuven Ramaty High Energy Solar Spectroscopic Imager (RHESSI) and other broad-band spectrometers.
\end{abstract}

Keywords Flares $\cdot$ Microflares and nanoflares

\section{Introduction}

A solar flare is a consequence of the rapid release of magnetic energy in the solar corona that can affect all layers of the solar atmosphere. Typical large flares can release up to $10^{32} \mathrm{ergs}$ of energy and are among the most violent solar phenomena. Much smaller flares, called micro- and nanoflares, with respective energy not larger that $10^{29} \mathrm{ergs}$, are very frequent and have been discussed widely, especially in the context of coronal heating mechanisms. Parker

\footnotetext{
$凶$ M. Gryciuk

mg@cbk.pan.wroc.pl

1 Space Research Centre, Polish Academy of Sciences, Bartycka 18A, 00-716 Warsaw, Poland

2 Astronomical Institute, University of Wroclaw, Kopernika 11, 51-622 Wroclaw, Poland
} 
(1988) originally proposed that coronal heating could be explained by numerous nanoflares, an idea later developed by Lee, Petrosian, and McTiernan (1995), Feldman, Doschek, and Klimchuk (1997) and Veronig et al. (2002). It appears that the total energy generated by nanoflares may be insufficient to explain the observed emission of the solar corona, although it is likely that more detailed statistical analysis is required to account for very small flares.

Catalogues of flare occurrences and characteristics represent very valuable resources that can provide us with more information as regards flare phenomena. An important problem is to collect a large sample of good quality data providing the parameters characterising flares in a coherent way. Flare timing and thermodynamic characteristics like temperature, emission measure and thermal energy content are of basic importance for studies of event physics and testing of models and processes of energy release in flares.

Timing data like flare start, peak, and end times have been systematically determined by the National Oceanic and Atmospheric Administration Agency (NOAA) for tens of thousands of solar flares using observations from the X-Ray Sensors (XRS: Garcia, 1994; Bornmann et al., 1996; Reinard et al., 2005) placed onboard the Geosynchronous Operational Environmental Satellites (GOES). The NOAA flare timing characteristics together with ancillary information are available online. ${ }^{1}$

Flare timing, as determined using the NOAA definition, may sometimes be misleading. In particular automatically determined times of event start and end may differ from the value obtained by visual inspection of X-ray light curves. For instance, the decay times of flares as determined from the NOAA procedure are systematically underestimated; times are often poorly determined for multi-peaked events; also, the majority of tiny events (with fluxes only slightly above the sensitivity threshold) cannot be properly characterised or are missing from the XRS database.

A more advanced solar flare detection algorithm for the interpretation of GOES 1-8 light curves was proposed by Aschwanden and Freeland (2012) (hereafter referred to as AF). Additional data reduction was needed in order to eliminate data gaps and spikes (the GOES flux shows instrument-related spikes that had to be removed before automatic analysis). The AF flare detection algorithm process reduced and smoothed data in order to search for local maxima and minima which represent potential flare start and end times. In the AF algorithm, it is also assumed that the event end time must occur before the start of a next flare (i.e. no overlapping events should exist). Additionally, a condition for minimum flare duration was set to be 1 minute.

AF claim that their algorithm is about 5 times more sensitive than the NOAA one. But even so, for many events, the start times and, in particular, end times do not agree with those estimated by visual inspection (see, for instance, Figure 1 of AF). Timing inaccuracies may be very large when flares overlap, which often occurs, even at times of low solar activity (see later). However, the advantage of the AF algorithm is that, besides the standard event characteristics provided by NOAA, the information on the value of the pre-flare background level is available for every flare.

Besides event timing, the other fundamental characteristics of basic importance for flares physics, are flaring plasma temperature $(T)$ and emission measure (EM). These are commonly derived by assuming that the emitting plasma is isothermal and using a ratio of fluxes measured in the two separate energy bands. The routine for $T$ and EM determination is straightforward but necessitates that the non-flaring plasma emission level is subtracted. Without subtracting this pre-flare background, the derived $T$ and EM time profiles often became unphysical (Bornmann, 1990; Ryan et al., 2012). Ryan et al. (2012) used Bornmann's

\footnotetext{
${ }^{1}$ See http://www.ngdc.noaa.gov/stp/solar/solarflares.html\#xray.
} 
pre-flare emission level concept to construct a so-called TEBBS algorithm (Temperature and Emission measure-Based Background Subtraction), which automatically corrects for an appropriate active region emission level based on the analysis of obtained temperature and emission measure behaviour throughout the event. The TEBBS-derived database of thermal flare plasma characteristics is available on the Solar Monitor web portal. ${ }^{2}$

In this paper, we give a different approach that allows flare characterisation to be obtained in a systematic and consistent way. The period of our research extends over the period of very low solar activity in 2009 between Cycles 23 and 24, when the level of solar X-ray radiation often fell below the XRS/GOES detection threshold. We use the excellent spectroscopic dataset recorded by the Solar Photometer in X-rays (SphinX: Sylwester et al., 2008, 2011, 2012; Gburek et al., 2011a,b, 2013; Kowalinski, 2012). The SphinX instrument had unprecedented time and energy resolution, high cadence and, what is particularly important, unprecedented sensitivity to observe the solar X-ray flux from the entire corona. The SphinX dataset covers nearly continuously the period from the end of February until the end of November 2009. The methods given are dedicated to the SphinX analysis of small flares, but they can be useful for events of any amplitude as observed by other instruments.

In Section 2 of this paper, we describe the SphinX spectrophotometer and its characteristics. Section 3 presents the methods used for the SphinX Flare Catalogue compilation: the flare detection algorithm (3.1), the model of the elementary flare time profile (3.2), and the background subtraction method and a procedure of optimum flaring plasma temperature and emission measure determination (3.3). In Section 4 a possible application of our model is given, and in Section 5 we summarise our results.

\section{SphinX Instrument and Data}

SphinX was the Polish spectrophotometer which observed solar soft X-ray emission in the energy range $1.2 \mathrm{keV}-15 \mathrm{keV}(0.8-10 \AA)$ in 256 energy bins with a time resolution of 1-5 sec (Sylwester et al., 2008, 2011, 2012; Gburek et al., 2011a,b, 2013; Kowalinski, 2012). The width of a single energy bin was about $59.2 \mathrm{eV}$ and the measured value of the spectral resolution (FWHM) was $464 \mathrm{eV}$. The instrument's field of view was $\approx 2$ degrees; thus the instrument covered the solar disk and low corona. SphinX operated onboard the Russian CORONAS-Photon satellite (Kotov, 2011) as a part of the Telescopic Spectroheligraphic Imaging System (TESIS: Kuzin et al., 2009, 2011). The spacecraft was launched on 30 January 2009 from Plesetsk Cosmodrome in northern Russia. CORONAS-Photon was put into a nearly circular low polar orbit with inclination 82.5 degrees, altitude 550 kilometers and period 96 min. SphinX was activated on 22 February and took measurements until 29 November, 2009 when the satellite mission was unexpectedly terminated.

In its orbit, the spacecraft regularly crossed regions of increased energetic particle density. In radiation belts and particularly in the South Atlantic Anomaly (SAA), SphinX measurements were strongly disturbed by additional contributions caused by the interaction of particles with the instrument and detectors. The particle signal in SphinX measurements must be removed from the data before a physical analysis of solar flux can be performed.

The presence of gaps in solar observations was caused by orbital nights when the spacecraft was in the Earth's shadow. These night periods lasted up to about half an hour per orbit. There were periods lasting two to three weeks when CORONAS-Photon was in continuous sunlight, so uninterrupted SphinX observations are available. On average, the duty

\footnotetext{
${ }^{2}$ See www.solarmonitor.org/TEBBS/.
} 


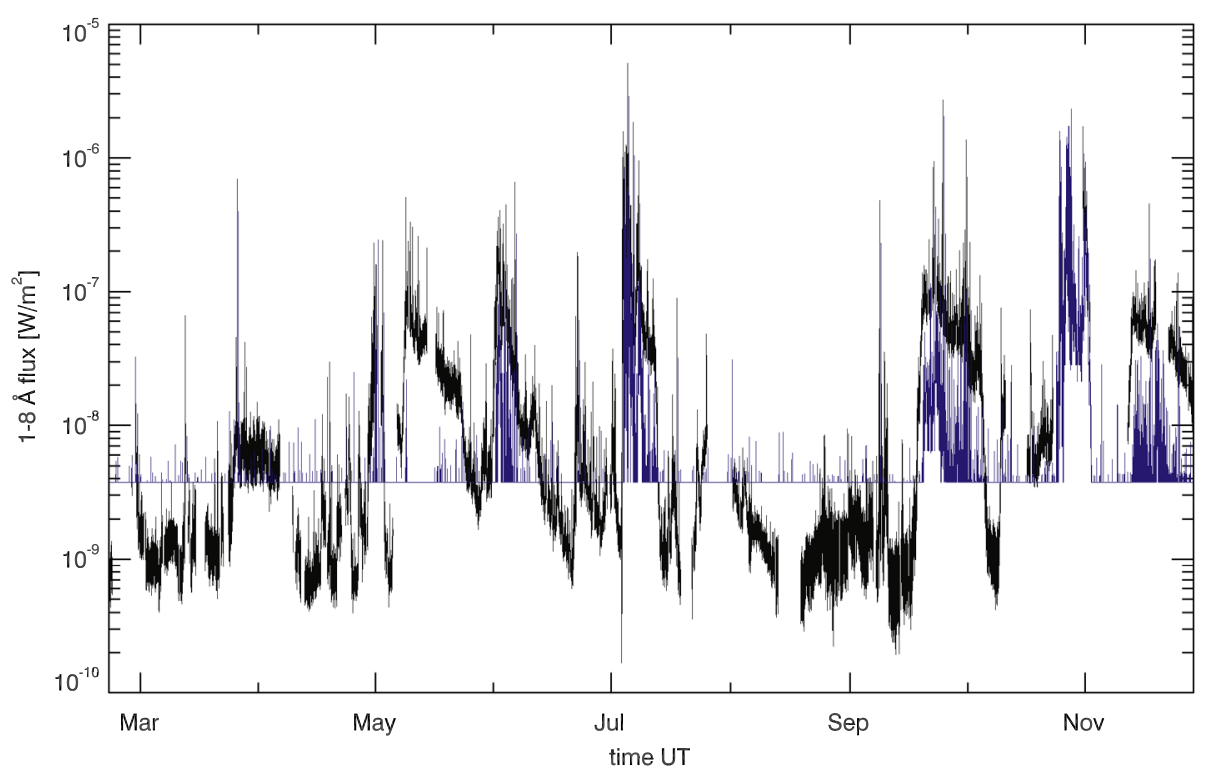

Figure 1 Solar fluxes in the 1-8 $\AA$ wavelength band derived from SphinX data (in black) compared with those observed by GOES (in blue). The characteristic flux increases are due to the presence of active regions and flares. The GOES sensitivity threshold is placed at the level of $3.73 \times 10^{-9} \mathrm{~W} / \mathrm{m}^{2}$.

cycle of SphinX for solar measurements was approximately $40 \%$. Promptly reduced data are stored in the SphinX Level 1 Data Catalogue available on the webpage of the Solar Physics Division, Space Research Centre of the Polish Academy of Sciences (SRC-PAS). ${ }^{3}$

SphinX measurements were made during 2009, a period of extremely low solar activity. At the time, SphinX was the only instrument capable of reliably measuring solar X-ray emission with high temporal and energy resolution. The SphinX instrument allowed the solar X-ray flux to be recorded at levels $\approx 100$ times below the detection threshold of the XRS detectors on GOES. Because of its high sensitivity, the SphinX instrument observed more than 1600 flares and brightenings over the nine months of its operation in 2009.

The SphinX detectors observed the solar soft X-ray emission in an energy range similar to that of GOES. The SphinX detectors observed solar X-ray emission in a band similar to that of GOES (1-8 Å range). To compare SphinX and GOES absolute fluxes (in $\mathrm{W} / \mathrm{m}^{2}$ ), a knowledge of the detector response matrix (DRM) is required as well as an assumption as regards the thermal emission of the emitting plasma. For the latter, we use the simple assumption that the plasma is isothermal, which we consider valid for the small-flare events observed and for the narrow energy range of the detector. The SphinX DRM is derived from pre-launch instrument calibration (Gburek et al., 2013). The SphinX fluxes are then determined by integrating photon energies in the range $1-8 \AA(1.5-12.4 \mathrm{keV})$. In Figure 1 , SphinX irradiances (in black) and GOES irradiances (in blue) (in $\mathrm{W} / \mathrm{m}^{2}$ ) are compared over the period February to November 2009. The GOES sensitivity limit $\left(3.73 \times 10^{-9} \mathrm{~W} / \mathrm{m}^{2}\right)$ is indicated, and the figure illustrates how much of this time period the solar X-ray flux was below this limit.

\footnotetext{
${ }^{3}$ See http://156.17.94.1/sphinx_I1_catalogue/SphinX_cat_main.html, or contact the author.
} 
The high sensitivity of SphinX detectors allows many tiny events to be observed at levels not seen before, below the GOES detection threshold. The strongest observed event was the C2.7 class flare on July 5, 2009.

The soft X-ray events with well pronounced maxima are categorised according to their intensities on the $1-8 \AA$ channel light curve. Every event is assigned a letter A, B, C, M and $\mathrm{X}$ representing flux values in units of $1.0 \times 10^{-8}, 1.0 \times 10^{-7}, 1.0 \times 10^{-6}, 1.0 \times 10^{-5}$ and $1.0 \times 10^{-4}$ (in W/ $/ \mathrm{m}^{2}$ ), respectively (Lang, 2001). For instance a flare labelled $\mathrm{C} 2.7$ has the maximum intensity of $2.7 \times 10^{-6} \mathrm{~W} / \mathrm{m}^{2}$. We propose the addition of two levels of X-ray emission, S ("small" $=10^{-9} \mathrm{~W} / \mathrm{m}^{2}$ ) and $\mathrm{Q}$ ("quiet" $=10^{-10} \mathrm{~W} / \mathrm{m}^{2}$ ), which are below the GOES sensitivity limit.

\section{Methodology for Compiling the SphinX Flare Catalogue}

\subsection{Detection of Flares from the Light Curve Analysis}

The first step in the analysis of the SphinX light curve is the detection of flare peaks. In order to process this large database (about $2 \mathrm{~GB}$ ), a semi-automatic procedure is devised. A semi-automatic method also enables a systematic event list to be compiled. Descriptions of several flare detection algorithms have been discussed elsewhere. The routines used for GOES (Aschwanden and Freeland, 2012) and Kepler (Brett, West, and Wheatley, 2004) can be given as examples. The recent paper by Ryan et al. (2016) discussed many aspects of flare-finding algorithms for the LYRA dataset. These algorithms took into account individual characteristics of particular observations and were dedicated to specific instruments. We find them difficult to apply to SphinX measurements, so an independent flare detection is devised instead.

Our algorithm uses SphinX flux spectrally integrated over the energy range 1.2 to $15 \mathrm{keV}$. The measurements are initially box-car averaged over the interval of $70 \mathrm{~s}$. This duration is chosen empirically in order to reduce statistical noise, while not losing time resolution necessary to recognise small brightenings. For such a re-sampled and smoothed dataset the algorithm searches for consecutive increases of four points $\left(t_{i}, t_{i+1}, t_{i+2}, t_{i+3}\right)$ satisfying the condition that the count rate value (CR) of the fourth point $\left(\mathrm{CR}\left(t_{i+3}\right)\right)$ is not lower than the initial value multiplied by 1.03 (i.e. $1.03 \times \mathrm{CR}\left(t_{i}\right)$ ). Next, the three consecutive decreasing flux points occurring after $t_{i+3}$, are searched for in the light curve. The time of flare maximum is defined as the global maximum between those two. This algorithm allows the peak times for most events to be identified. However, not all events are automatically detected. We notice that by running the algorithm on time-reversed series of the measurements, additional flare peaks are detected. Flux maxima found using forward and backward searching methods are included in the SphinX flare maxima timing list.

An illustration of the flare-peak detecting algorithm is shown in Figure 2, where we give an example of the SphinX light curve in the entire spectral range $(1.2-15 \mathrm{keV})$ with a series of events observed on 29 and 30 April, 2009. As can be seen, the automatic algorithm can detect flare maxima in most cases but still cannot identify some events which are obvious by eye inspection. In addition some events are missed because of data gaps present in the SphinX light curve. On the other hand, the algorithm seems to be "oversensitive" in some cases and detects tiny features which are not evident by eye inspection. That is why in the final stage we make a visual inspection and correct misidentifications.

As a result of automatic searching, we find about two thousand events over the SphinX operation period. Due to the algorithm oversensitivity, many noise features are detected and 


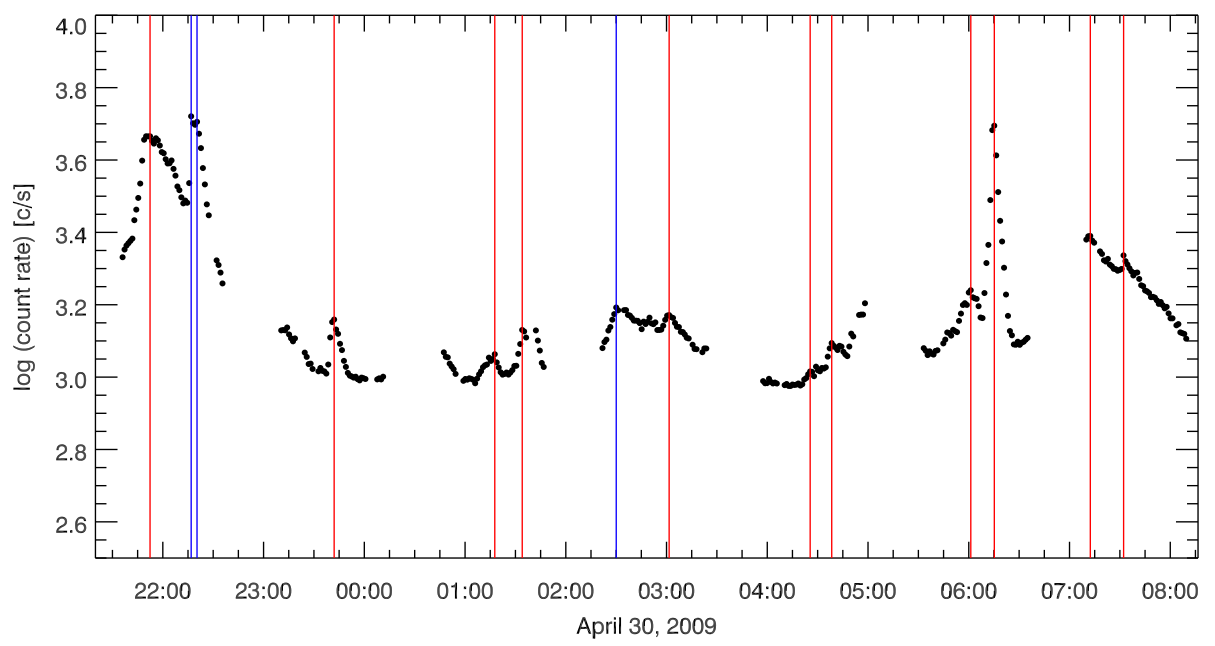

Figure 2 An example of a SphinX 1.2-15 keV light curve during 11 hours of observations with flares detected by the semi-automatic algorithm marked. Automatically detected times of flare maxima are indicated with red vertical lines. Maxima added by subsequent visual inspection are shown as blue lines in the plot.

during visual inspection we reject almost 500 such events. In the process of inspection by eye, about 300 flares are added. The resulting list of detected events contains times of event maxima. Overall, 1604 flares or flare-like events are identified in the SphinX detector D1 light curve.

The light-curve algorithm allows us to create a list of peak times of SphinX-observed events covering the period of the deep minimum of solar activity in 2009. The list and full catalogue can be found on the webpage of the Solar Physics Division SRC-PAS. ${ }^{4}$ The Catalogue not only contains the time of maximum occurrence but also other flare characteristics. Some of these characteristics, including the flare start and end times, are obtained with the methods described in the next sections of this paper.

\subsection{The Concept of Elementary Flare Profile}

In order to interpret individual flare brightenings observed on the SphinX light curve, we used a simple analytical formula (Equation (1)) that defines a so-called elementary ${ }^{5}$ soft X-ray flare time profile (EFP). Fitting the profile to observations allows us to easily parametrise a simple, single-peaked events and to decompose complex, multi-peaked flares into a series of elementary events. Despite the fact that SphinX was operating during a period of very low solar activity, in many cases events overlapped.

With the concept of an EFP, we relate the shape of the observed light curve to the time profile of energy release. The energy liberated at a given time is assumed to be released according to a Gaussian profile and instantly monotonically dissipated. If we assume that

\footnotetext{
${ }^{4}$ See http://www.cbk.pan.wroc.pl/mg/SphinX_fl.html, or contact the author.

5 The term "elementary" in the context of temporal flare profiles was originally introduced by van Beek, de Feiter, and de Jager (1974) to describe hard X-ray flares. Van Beek and co-authors found that hard X-ray flares can be decomposed into a number of smaller spikes, and called these spikes "Elementary Flare Bursts". In this work we use term "elementary" to describe a simple flare time profile as seen in soft X-rays.
} 
Figure 3 The model of the soft X-ray flare time profile $f(t)$ results from the convolution of a Gaussian function describing the energy release rate $g(x)$ (Equation (2)) with an exponential decay function $h(x)$ describing energy dissipation (Equation (3)).

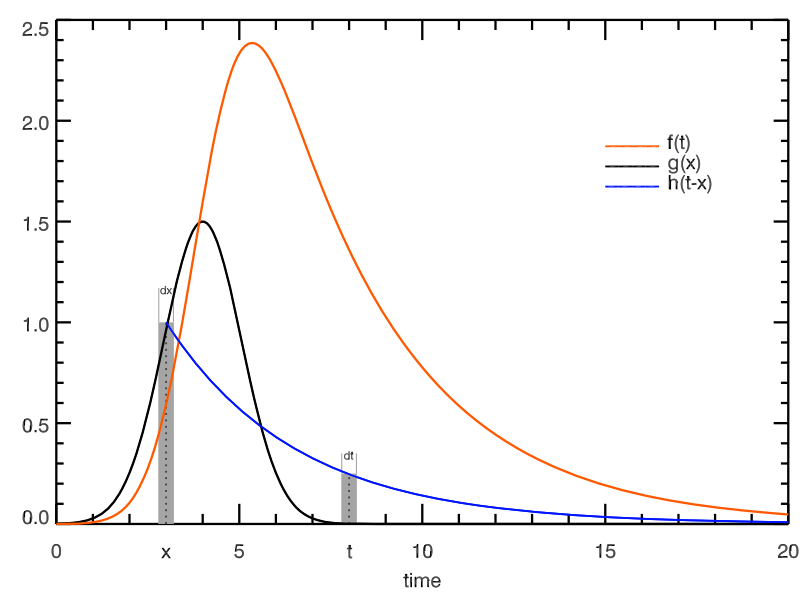

release and deposition of energy during a flare is described by a function $g(x)$ and dissipation of released energy by a monotonically decreasing function $h(x)$, then the energy released during an interval $\mathrm{d} x$ around the time $x$ is $g(x) \mathrm{d} x$. That energy undergoes dissipation (by radiation and conduction processes) and at the moment $t$ (following $x$ ) the available energy $\mathrm{d} f(t)$ is $g(x) h(t-x) \mathrm{d} x$. The total energy $f(t)$ available at time $t$ is the integral of $\mathrm{d} f$ from 0 to $t$.

We can express the overall event time profile $f(t)$ as the convolution of two profiles $g(x)$ and $h(x)$ defined for positive $x$ arguments as follows:

$$
f(t)=\int_{0}^{t} g(x) h(t-x) \mathrm{d} x .
$$

The mathematical form of the heating function can be complicated, but for convenience and simplicity we use the Gaussian form:

$$
g(x)=A \exp \left(-(x-B)^{2} / C^{2}\right)
$$

while the dissipation function describing processes of energy loss is expressed by an exponential decay with $h(0)=1$ :

$$
h(x)=\exp (-D x)
$$

where $A, B, C, D$ are shape parameters and $t$ is the time.

In our model, the SXR flare time profile reflects the common action of two simultaneous processes: energy release and loss. The energy release process is assumed to have a Gaussian form (see e.g. Aschwanden, Dennis, and Benz, 1998), while the energy dissipation rate is assumed to have an exponential form (see e.g. Aschwanden and Freeland, 2012). The parameter $D$ in Equation (3) corresponds to the reciprocal of the cooling time. In Figure 3 we plot the $g(x)$ and $h(x)$ functions as well as the convolution $f(t)$.

After incorporating Equations (2) and (3) into Equation (1) and integrating, the EFP profile can be presented in a form suitable for numerical calculation and is expressed by

$$
f(t)=\frac{1}{2} \sqrt{\pi} A C \exp \left[D(B-t)+\frac{C^{2} D^{2}}{4}\right]\left[\operatorname{erf}(Z)-\operatorname{erf}\left(Z-\frac{t}{C}\right)\right],
$$




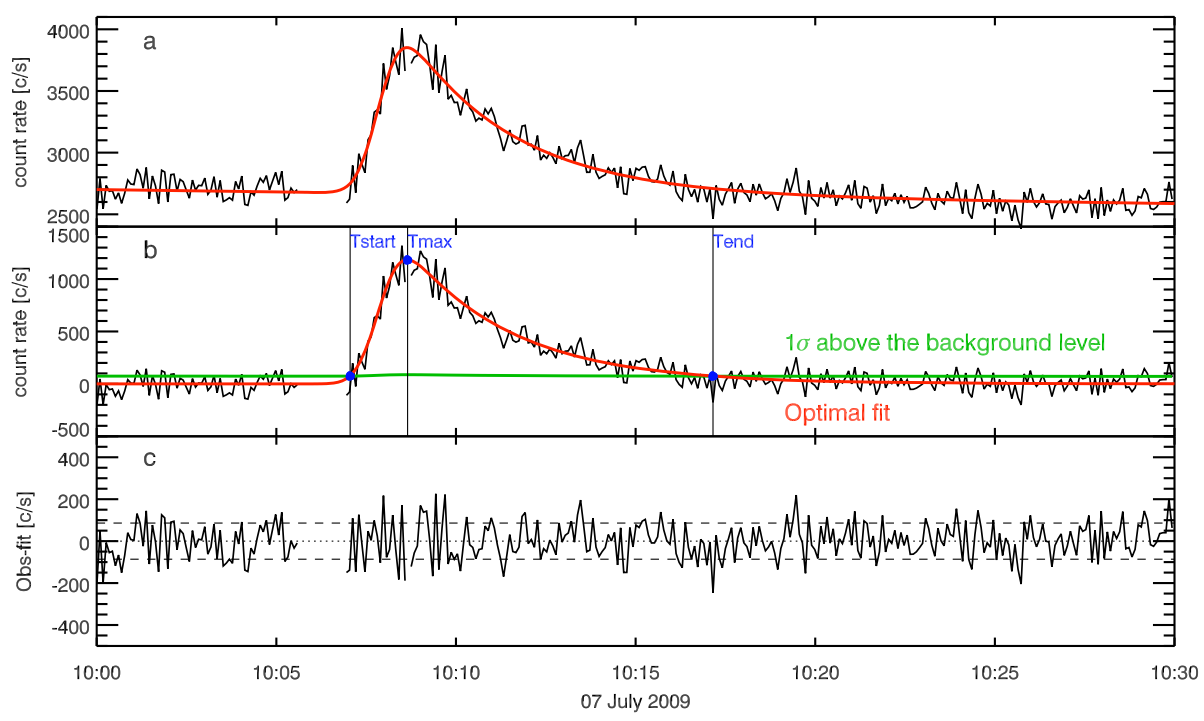

Figure 4 An example of a SphinX light curve (black line) as observed in the energy range $1.2-15 \mathrm{keV}$ before (panel a) and after (panel b) background subtraction with the best quality fit of the elementary flare profile (red curve) overplotted. Times of the start, maximum and the end times are shown by straight vertical lines. The horizontal green line indicates the level of $1 \sigma$ above the fitted background. The crossing points of this line with the fitted flare profile are used for the determination of flare start and end times. Panel c shows the residuals of the fit, with levels of $\pm \sigma$ marked by dashed lines.

where

$$
Z=\frac{2 B+C^{2} D}{2 C}
$$

and erf is the error function, defined as $\operatorname{erf}(t)=\frac{2}{\sqrt{\pi}} \int_{0}^{t} \exp \left(-s^{2}\right) \mathrm{d} s$. The error function is widely used in statistics and is thus available in many programming packages for symbolic and numerical evaluation. It is also available in the IDL software environment used for the present study.

We assume that the background SXR emission on which the flare contribution is superimposed has a linear form:

$$
b(t)=E t+F,
$$

with two free parameters $E$ and $F$.

The complete formula that represents the observed flare light curve $\operatorname{lc}(t)$ depends on the value of the six parameters $(A, B, C, D$ : flare shape and $E, F$ : linear background).

For fitting the elementary flare time profile to flare profiles identified in SphinX light curves, we used the MPFITFUN (Markwardt, 2009) procedure, which is published and available. $^{6}$ The MPFITFUN procedure is translated from the MINPACK-1 package (More, 1977) for solving nonlinear equations and nonlinear least squares problems. We find MPFITFUN faster, flexible, and of improved performance in comparison to the generic routine CURVEFIT available within the IDL environment. The size of the window around the flare used to fit the EFP model is determined manually for each event independently. We select

\footnotetext{
${ }^{6}$ See http://www.physics.wisc.edu/ craigm/idl/mpfittut.html.
} 


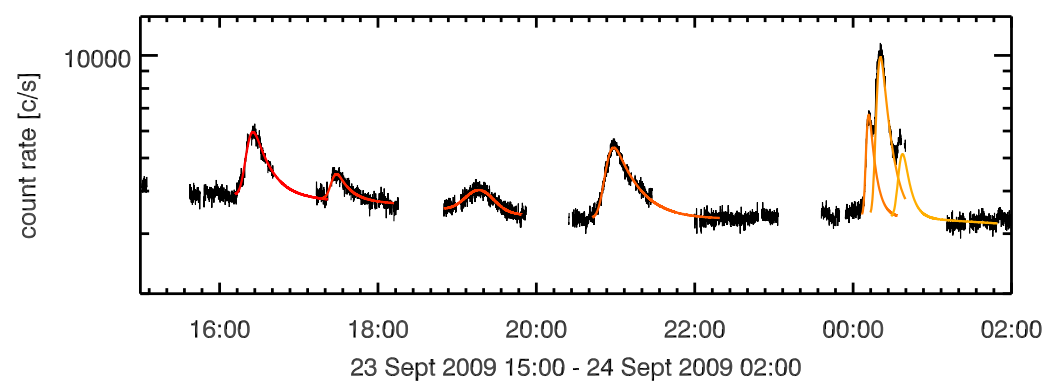

Figure 5 SphinX 1.2-15 keV light curves with optimal fits of elementary flare profiles in colour (Equation (4)). The last three events in the plot contribute to the multi-peaked event. The fitted profiles are plotted from the start to the end time of the respective flares (see text for details).

intervals which are as long as possible, to include as much non-flaring background as possible.

We use optimally fitted EFPs to determine a reliable timing of the event i.e. start, maximum and, end times as well as the flare magnitude (the event amplitude after background subtraction). The start time of the event is set to correspond to the time when the fitted flare profile (without the background) increased to the level of one standard deviation value $(1 \sigma)$ of the background. The end time is defined as the instant when the fitted profile crossed the $1 \sigma$ level on the flare decay. To estimate the standard deviation of the SphinX background we select 318 time intervals of the light curve with constant background and we calculate standard deviations for each interval. As a result we find the relation between the count rate and the estimated standard deviation values $\left(\sigma=(0.12 \pm 0.002\right.$ counts $/ \mathrm{s}) \mathrm{CR}^{(0.83 \pm 0.002)}-$ $(6.93 \pm 0.153$ counts $/ \mathrm{s}))$. Using this relation we are able to automatically detect timing parameters of flares. In Figure 4 we present an example of a flare light curve observed by SphinX on 07 July 2009 at 10:08 UT together with the best quality fit (red curve). The green line indicates the level of $1 \sigma$ above the background. Times of the start and of the end of the flare are set at the points of time where the fitted flare profile crossed this line.

As mentioned, it is possible to fit complex multi-peaked profiles by combining a series of individual elementary profiles, in cases when it is not possible to accommodate the observed profile with an optimum single EFP fit. How many elementary profiles are fitted to a complex event depends on the number of discernible maxima and the resulting profile. In our analysis, we fit to the light curve as many EFP profiles as necessary to obtain residuals at a $1 \sigma$ level above the local background.

Figure 5 shows an example giving a light curve recorded by SphinX (black line) on 23-24 September 2009. The optimum quality fits of elementary flare profiles are shown in colour. The last three profiles in the plot represent an example of decomposition of multiple EFPs contributing to multi-peaked event. An example of EFP fits to a complex event is shown in Figure 6 together with the plot of residuals.

\subsection{Background Subtraction Method and Flaring Plasma Diagnostics}

It is well known that, as the flare progresses, the average temperature and emission measure increases to a certain maximum level and then declines during the decay phase (Jakimiec et al., 1992; Reale, Peres, and Orlando, 2001). This general trend is not dependent on the flare strength (Sterling, Doschek, and Pike, 1994; Feldman et al., 1995; Feldman, Doschek, and Behring, 1996). 


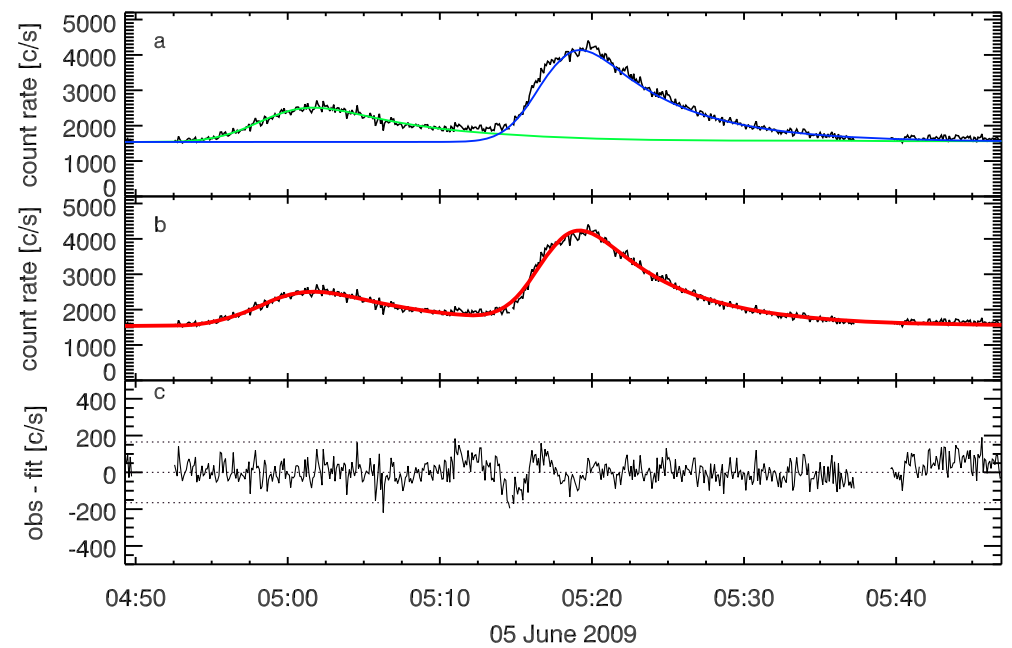

Figure 6 Panel (a): Example of double-peaked SphinX light curve recorded on 05 June 2009 in the energy range 1.2-15 keV (black line) together with two elementary flare profile fits (green and blue lines). Red line in panel (b) indicates summed profile of two fitted EFPs. Residuals obtained by subtracting the summed profiles from the data are shown in panel (c). Dotted lines indicate $\pm \sigma$ levels.

Figure 7 Logarithm of the ratio of observed SphinX light curves in two energy bands as a function of effective plasma temperature.

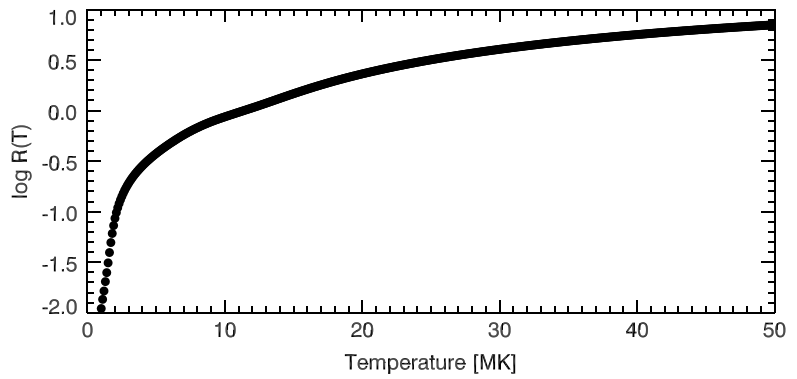

As Bornmann (1990) showed, observed flare flux includes a quiescent contribution due to the non-flare emission from the flaring plasma referred to here as "background". Subtraction of an incorrect background corresponding to the total pre-flare flux results in derived temperatures and/or emission measures (in the isothermal approximation) that disagree with physically acceptable trends.

We correct SphinX light curves for the background using similar methods to the TEBBS algorithm mentioned previously. However, instead of taking a constant background (as used in the original TEBBS method) we assume a background with time dependence having a fixed slope (the parameter $E$ in Equation (5)).

Flaring plasma diagnostics for SphinX events are determined using the flux-ratio method similar to that commonly used for interpretation of GOES X-ray measurements. The application of the method for XRS/GOES data was described by Thomas, Crannell, and Starr (1985) and updated by White, Thomas, and Schwartz (2005). Narukage et al. (2011) used this method for determination of $T$ and EM from the $X$-ray telescope (XRT)/Hinode observations. In the case of SphinX data analysis, a theoretical temperature response function is determined from the CHIANTI (version 6.0.1) atomic code (Dere et al., 1997; Landi and 


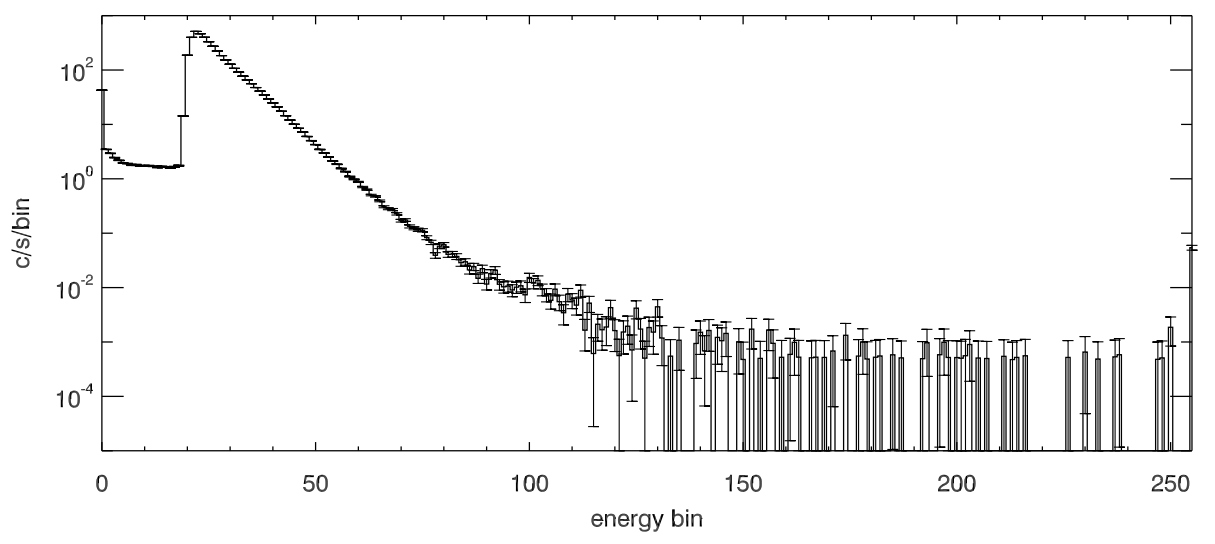

Figure 8 An example of a spectrum obtained by SphinX with error bars. The spectrum is averaged over an 11-hour time interval from 15:00 (23.09.2009) to 02:00 (24.09.2009). SphinX is able to measure the spectra of the entire solar corona in 256 energy bins between 1.2 and $15 \mathrm{keV}$.

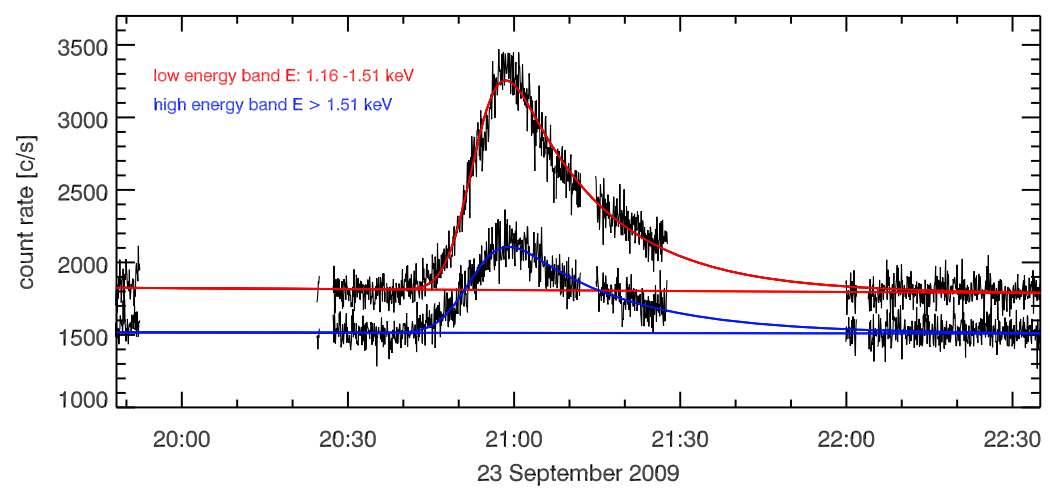

Figure 9 The SphinX light curves as observed in the two energy bands. Overplotted in colour are the best-fit elementary profiles including their respective backgrounds for the A4 class flare observed on 23 September 2009 at 20:58 UT. Dashed lines indicate optimal background levels for both bands.

Phillips, 2006) using a coronal set of elemental abundances from Feldman et al. (1992) (sun_coronal_feldman_1992_ext.abund) and ionisation equilibrium values from Mazzotta et al. (1998). The differences in the SphinX response functions based on CHIANTI versions 6.0.1 and 8.0.1 (available now in SolarSoft) are imperceptible. We have checked that the results obtained using other ionisation equilibria are negligible. This is in accordance with the previous conclusions from Kepa et al. (2005) for calculations of differential emission measure in the soft X-ray flaring plasma. The value of electron density is assumed to be $10^{10} \mathrm{~cm}^{-3}$, but for optically thin plasma (solar corona) the spectral line fluxes in the SphinX range are independent of density (Mewe and Schrijver, 1978). In Figure 7 we give the theoretical temperature response function derived for the SphinX instrument.

In our analysis we divide the observed flux into two energy bands spanning the ranges $1.16-1.51 \mathrm{keV}$ (low energy band) and above $1.51 \mathrm{keV}$ (high energy band). Below $1.16 \mathrm{keV}$ there is a strong decrease of SphinX sensitivity and the measured signal is dominated by 


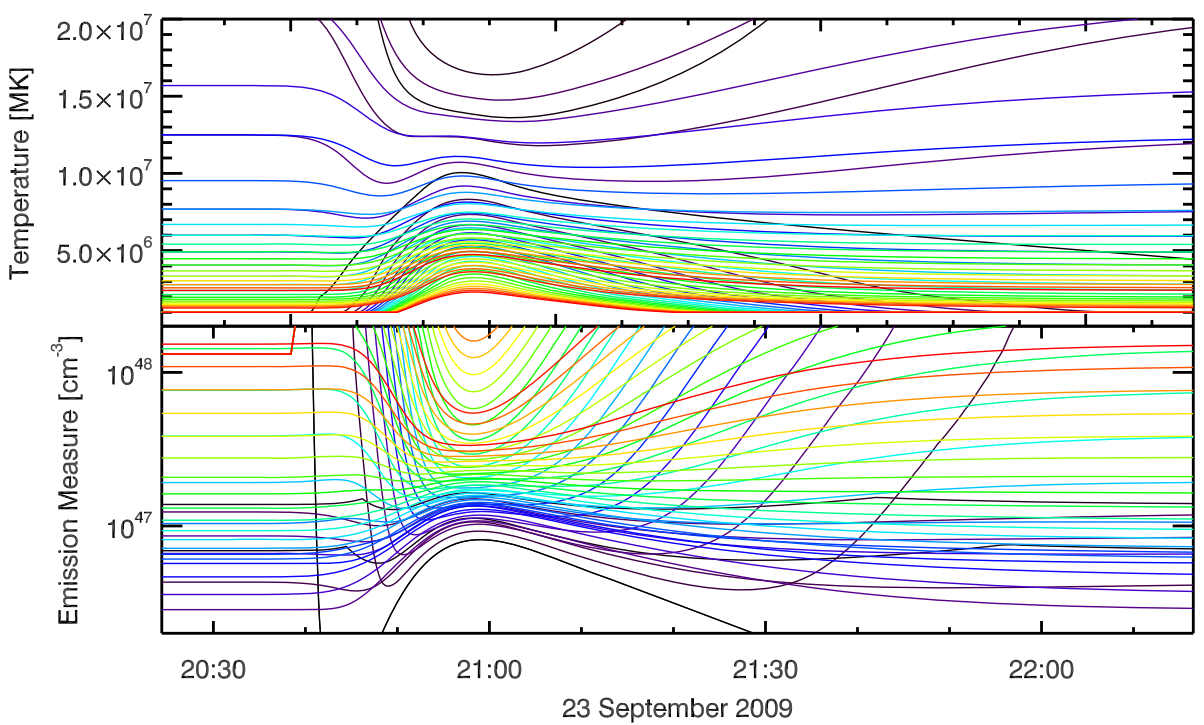

Figure 10 An example of families of $T$ (top panel) and EM (bottom panel) time profiles for different assumed background levels. For better visibility, we show only 57 out of 400 calculated profiles. Individual colours indicate different assumed background value combinations (20 levels for every energy band).

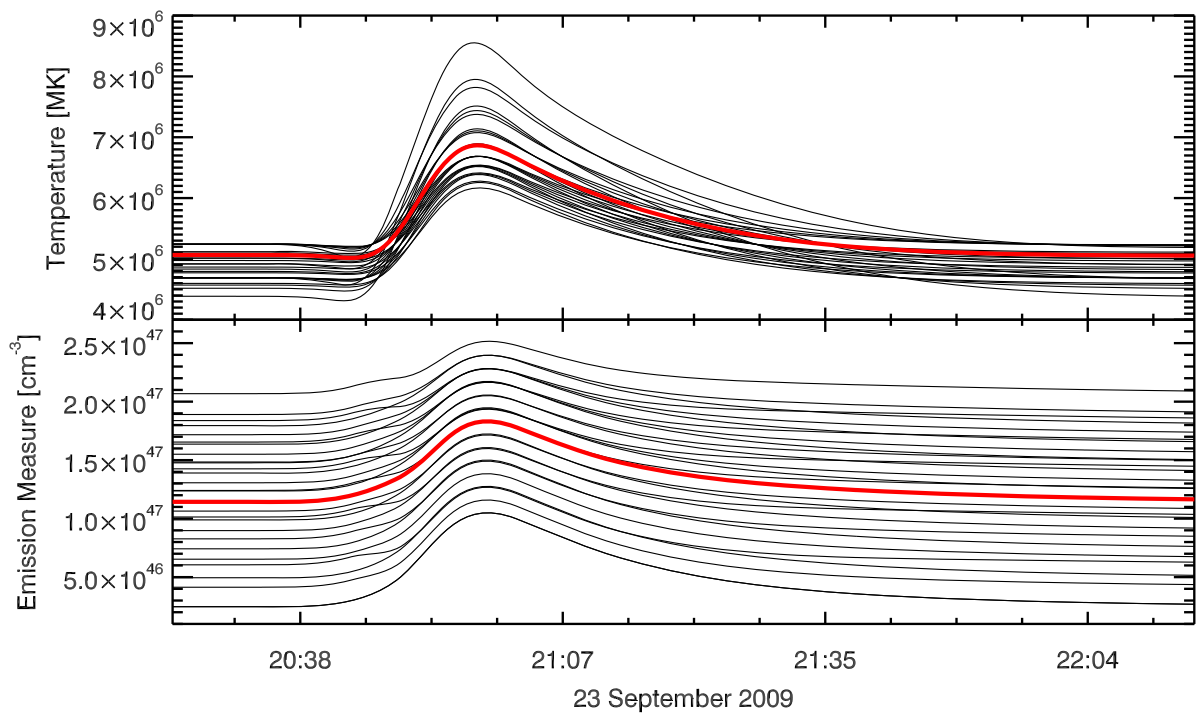

Figure 11 The set of $27 T$ and EM time profiles selected as acceptable. The thick red lines indicate our final selection.

electronic noise (Figure 8). By setting the energy between the two bands at $1.51 \mathrm{keV}$, we ensured good count statistics in both bands for various flare conditions (Engell et al., 2011).

Separate fits are made as described for low and high band light curves using the elementary flare profile described in Section 3.2 (Equations (4) and (5)). An example of a light 
curves for the flare observed by SphinX on 23 September 2009 (maximum at 20:58 UT) is shown in Figure 9. The figure also shows the best quality fit of the elementary flare time profile to light curves obtained in the two bands with corresponding linear optimum backgrounds levels.

As in the TEBBS method, for both SphinX channels, a set of 20 equally separated values of the $F$ parameter describing the background level (Equation (5)) are selected. All the values of $F$ are in the range from zero to the pre-flare background in each channel. We do not vary the slope of the background and assume the value of $E$ to be that from fitting Equation (5) to the observed flare light curve in each energy band. We next use the flux-ratio method to determine $T$ and EM profiles for all selected background levels. In this way, we obtain profiles for 400 different background combinations for both bands, separated for each elementary flare. Complex, multi-peaked flares are decomposed into a series of elementary components which are investigated one by one.

Many of the obtained $T$ and EM time profile solutions do not follow trends expected for flares (see Figure 10) and have to be rejected. We treat situations where the estimated temperature and emission measure fail to fit a standard pattern as unphysical (i.e. there is no single significant maxima near the time of the event peak). Additionally, we reject profiles showing any local maxima during their decay. The rejections limit the number of cases with acceptable time behaviour to a set of typically several tens for every investigated flare. In Figure 11 we give such a set of acceptable profiles.

From this set, we determine a final set of profiles. Different methods can be used for this final selection. We assume that the average of all acceptable EM profiles represents the most probable one, so we calculate the median value of all maxima over the time values of the $T$ profiles, and then we select the one with value closest to the calculated median. The final $T$ profile interchangeably indicates the background level position of both bands. The selected most probable $T$ and EM time profiles are marked with thicker red lines in Figure 11.

Our plasma-diagnostics method is based on the TEBBS algorithm, although in our analysis we use an elementary flare profile, not the originally observed light curve. Our approach therefore allows for the determination of thermal parameters of very weak events with better precision than the TEBBS method. Our method is also suitable for independent analysis of multi-peaked flares.

\section{An Example Application of EFP Light Curve Analysis}

The results of the flare plasma diagnostics obtained using fitting of EFPs to both energy channels make it possible to deduce flare heating/cooling properties. For this purpose, diagnostic diagrams (DD) can be used, as introduced by Jakimiec et al. (1992) based on the results of hydrodynamic modelling of the flaring loops. A number of flare models calculated by means of the Palermo-Harvard hydrodynamic code (Peres et al., 1982) are considered and the evolution of basic thermodynamic parameters of a flaring plasma are analysed in a $\log -\log$ density-temperature $(N-T)$ diagram. We find that using the $N-T$ diagrams, one can investigate the important physical quantity, viz. the volumetric heating function $E_{\mathrm{H}}(t)$, so they are called diagnostic diagrams. The two limiting branches during the cooling phase on the DD correspond to the abrupt switch off of the heating (the steep curve with inclination $\approx 2.0$ ) and to the sufficiently quasi-steady slow decrease of heating when the RTV scaling laws (Rosner, Tucker, and Vaiana, 1978) are fulfilled (a so-called quasi-steady-state, QSS, branch with inclination $\approx 0.5$ ). The evolution is intermediate between these two cases, and 

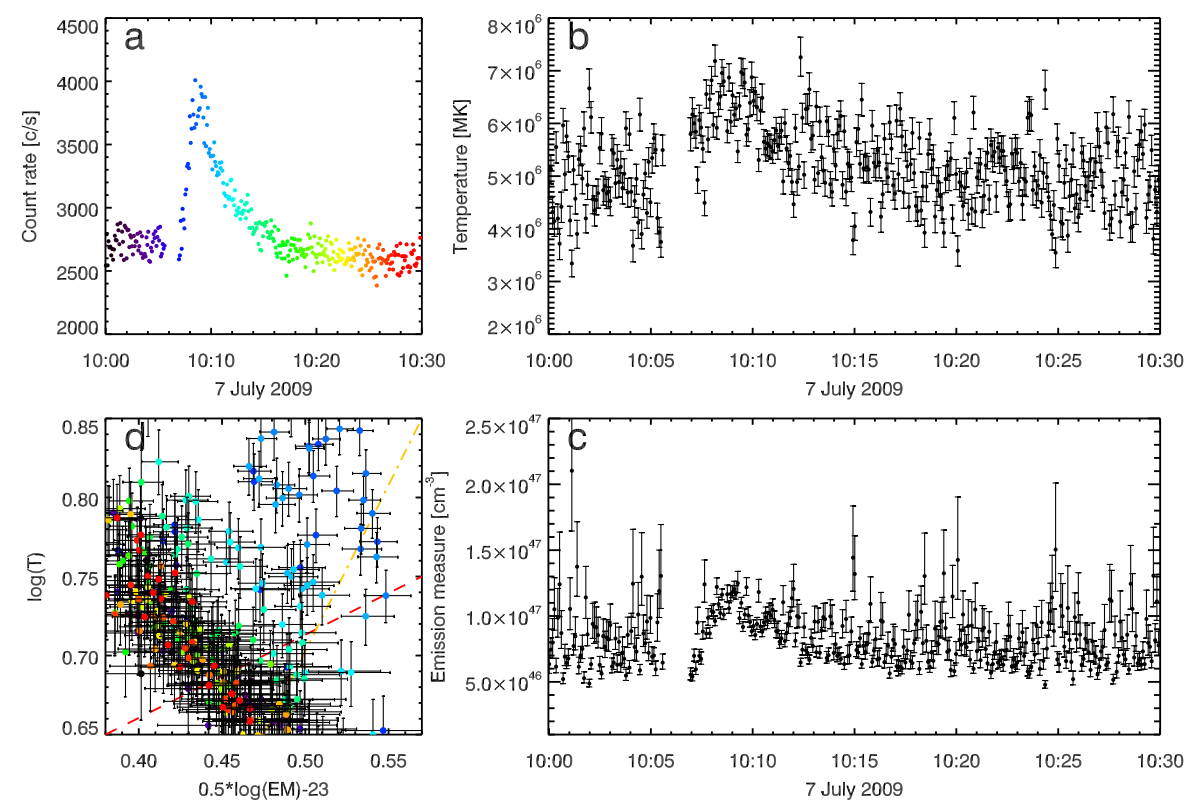

Figure 12 An example of flare plasma diagnostics based on the light curve of a flare observed by SphinX on 23 September 2009. Panel a shows the SphinX light curve (colour line) as observed in the energy range 1.2-15 keV. Panels b and c show estimated $T$ and EM time profiles calculated using the background-subtracted isothermal approximation method. Panel d shows the diagnostic diagram for the estimated $T$ and EM profiles. The red and yellow lines in the DD plot indicate two limiting cases: quasi-steady state and instant switching off of the heating, respectively.
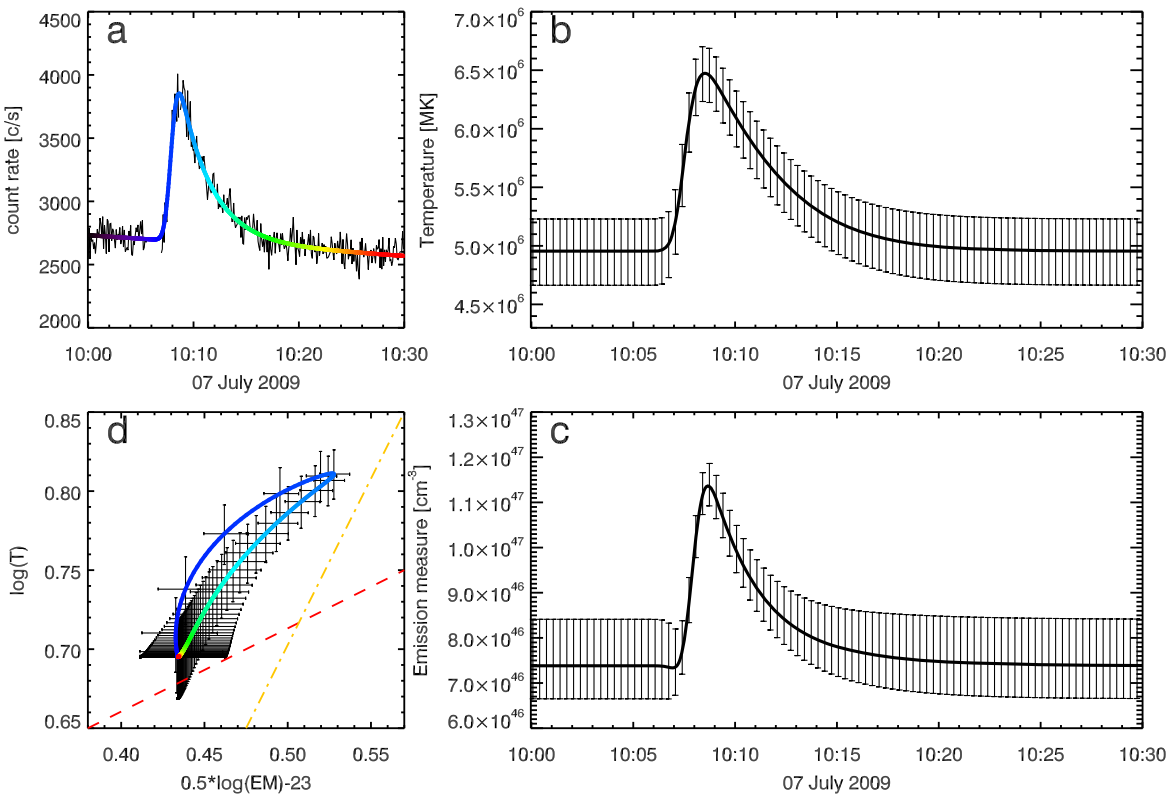

Figure 13 Same as Figure 10, but showing results for the best quality fit of the elementary flare profile including the background (colour line in panel a). 

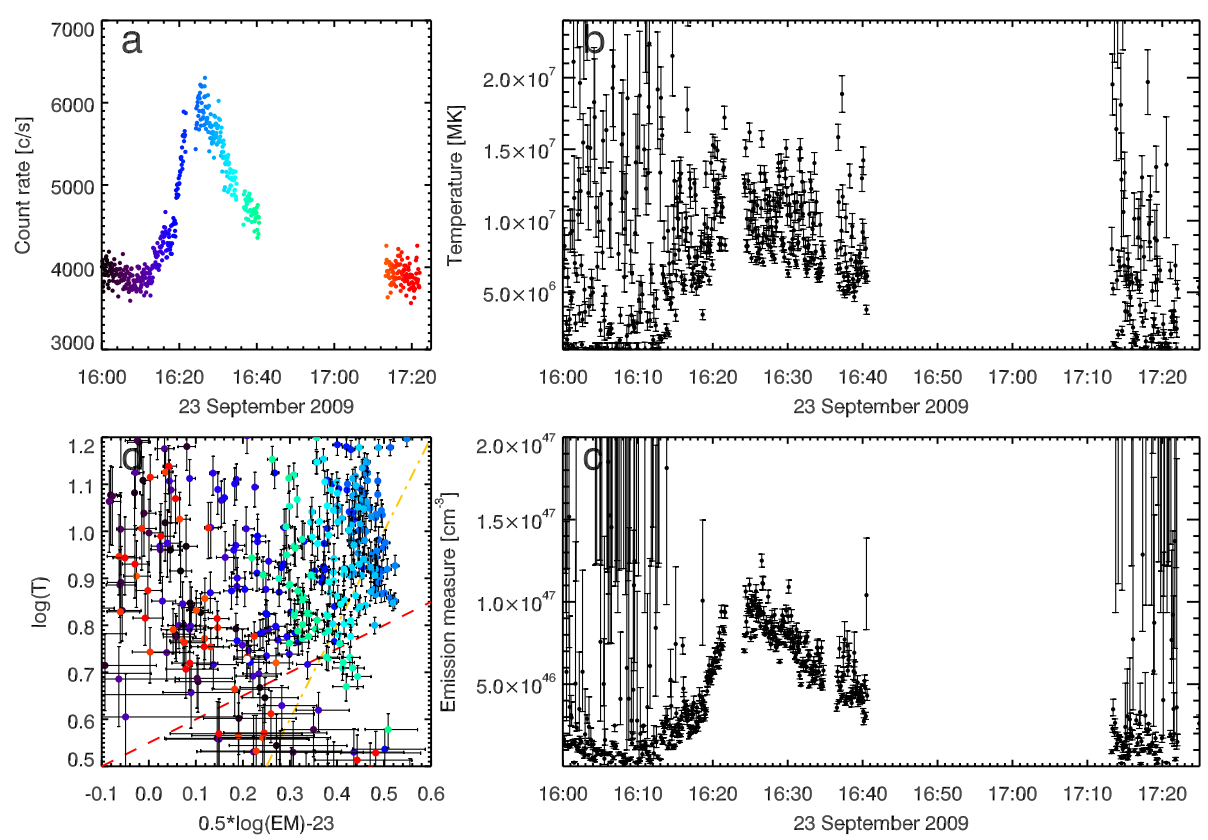

Figure 14 An example of flare plasma diagnostics based on the light curve of a flare observed by SphinX on 23 September 2009. Panel a presents the SphinX light curve (colour dots) as registered in the energy range 1.2-15 keV. Panels b and c show estimated $T$ and EM time profiles calculated using the presented EFP fit and background-subtracted isothermal approximation method. Panel d contains the diagnostic diagram for the estimated $T$ and EM values. The red and yellow lines in the diagnostic diagram (DD) plot indicate two limiting cases: quasi-steady state and instant switching off of the heating, respectively.

it corresponds to the decreasing heating with the e-folding decay time $\tau$ :

$$
E_{\mathrm{H}}(t) \sim \exp (-t / \tau)
$$

With the simplifying assumption that the emitting volume is constant, the $N-T$ diagrams can be successfully used to interpret the solar flare soft X-ray observations.

To illustrate the application of the EFP model for determination of $T$ and EM profiles we give example results for two B-class flares recorded by SphinX on 7 July and 23 September 2009. Figures 12 and 14 show light curves and time profiles of $T$ and EM, as obtained from light curves, and an empirical diagnostic diagram constructed for the parameters. Figures 13 and 15 show the same light curves, but with the best quality fit to the elementary flare profile overplotted. The time profiles of $T$ and EM, and the DD diagram given here are obtained using the fitted profiles. The results show the advantage of using the fitted EFP model against data points as observed. There is a relatively large variation of $T$ and EM at the beginning and late phases, especially for small events. This makes the interpretation of the results more difficult. The DD diagrams show how the EFP model method described here can be a useful tool in small-flare analysis.

Temperature and emission measure as obtained by the proposed method could also be used for more precise determination of the thermal energy of flares (de Jager et al., 1989). Based on the obtained $T$ and EM maximal values and images recorded by the GOES Soft $X$-ray Imager (SXI: Lemen et al., 2004; Pizzo et al., 2005) it is possible to determine the 

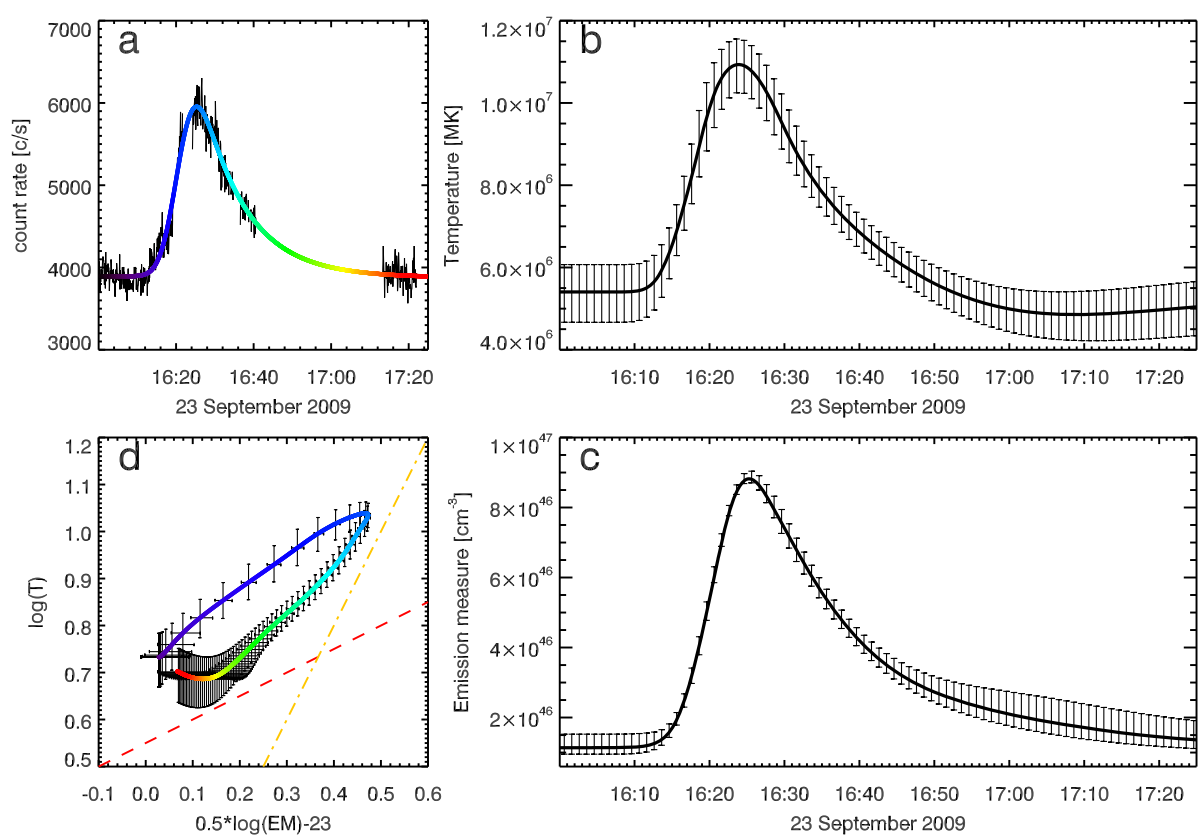

Figure 15 Same as Figure 12, but here the results for the best quality fit of the elementary flare profile including the background (colour line in panel a) are given.

thermal energy of one of the 23 September 2009 flares. In our calculation, we assume a unit filling factor. An emission volume is inferred from the SXI emission size source within a $50 \%$ isophote. The estimated thermal energy for this case equals $\approx 5.2 \times 10^{29} \mathrm{ergs}$.

\section{Summary}

As a result of running the flare detection algorithm on light curves from the SphinX instrument, we obtain a list of flare maxima. Start and end times as well as corrected flare maximum times are then determined using fits of the formulae given in the text to the detected flare light curves. By using a model of an elementary flare profile (EFP) it is possible to decompose complicated multi-peaked events into a number of elementary energy release components. Performing light-curve decomposition allow us to determine the timing of particular SphinX-observed events. The elementary flare profiles allow us to investigate the $T$ and EM behaviour during flares more satisfactorily. This is of particular importance for investigations of small flares where statistical fluctuations of the signal are relatively large. The method used for $T$ and EM estimation allows us also to determine a time-dependent linear background.

The algorithms described here can obtain systematic and consistent flare characterisations. Our final goal is the preparation of a comprehensive catalogue of flare events and brightenings observed by SphinX during the 2009 solar minimum. The SphinX Flare Catalogue includes all flares detected by the algorithm and their characteristic parameters. Using the methods described, the following flare parameters are listed in the catalogue: flare start, maximum, and end times, class as determined from flare amplitude, six parameters describing the EFP model, maximum temperature and emission measure. We plan to supplement the 
catalogue with time profiles of $T$ and EM and diagnostic diagrams of particular flares. Additional information from X-ray measurements of other solar instruments like XRT/Hinode and GOES will also be provided if available.

The SphinX Flare Catalogue, which is published on the SRC-PAS Solar Physics Division webpage and which will be systematically updated, will be used for further analyses by statistical methods.

Acknowledgements The research leading to these results has been supported by Polish National Science Centre grant No. DEC-2013/09/N/ST9/02209. We also thank an anonymous referee for constructive comments, which have improved this article. We are also indebted to K.J.H. Phillips for improving the English.

Disclosure of Potential Conflict of Interest The authors declare that they have no conflict of interest.

Open Access This article is distributed under the terms of the Creative Commons Attribution 4.0 International License (http://creativecommons.org/licenses/by/4.0/), which permits unrestricted use, distribution, and reproduction in any medium, provided you give appropriate credit to the original author(s) and the source, provide a link to the Creative Commons license, and indicate if changes were made.

\section{References}

Aschwanden, M.J., Freeland, S.L.: 2012, Automated solar flare statistics in soft X-rays over 37 years of GOES observations: The invariance of self-organized criticality during three solar cycles. Astrophys. $J$. 754, 112. DOI.

Aschwanden, M.J., Dennis, B.R., Benz, A.O.: 1998, Logistic avalanche processes, elementary time structures, and frequency distributions in solar flares. Astrophys. J. 497, 972. DOI. ADS.

Bornmann, P.L.: 1990, Limits to derived flare properties using estimates for the background fluxes - examples from GOES. Astrophys. J. 356, 733. DOI.

Bornmann, P.L., Speich, D., Hirman, J., Pizzo, V.J., Grubb, R., Balch, C., Heckman, G.: 1996, GOES solar $\mathrm{X}$-ray imager: overview and operational goals. 2812, 309. DOI.

Brett, D.R., West, R.G., Wheatley, P.J.: 2004, The automated classification of astronomical light curves using Kohonen self-organizing maps. Mon. Not. Roy. Astron. Soc. 353, 369. DOI.

de Jager, C., Bruner, M.E., Crannel, C.J., Dennis, B.R., Lemen, J.R., Martin, S.F.: 1989 In: Energetic Phenomena on the Sun.

Dere, K.P., Landi, E., Mason, H.E., Monsignori Fossi, B.C., Young, P.R.: 1997, CHIANTI - an atomic database for emission lines. Astron. Astrophys. Suppl. 125, 149. DOI. ADS.

Engell, A.J., Siarkowski, M., Gryciuk, M., Sylwester, J., Sylwester, B., Golub, L., Korreck, K., Cirtain, J.: 2011, Flares and their underlying magnetic complexity. Astrophys. J. 726, 12. DOI.

Feldman, U., Doschek, G.A., Behring, W.E.: 1996, Electron temperature and emission measure determinations of very faint solar flares. Astrophys. J. 461, 465. DOI. ADS.

Feldman, U., Doschek, G.A., Klimchuk, J.A.: 1997, The occurrence rate of soft X-ray flares as a function of solar activity. Astrophys. J. 474, 511. ADS.

Feldman, U., Mandelbaum, P., Seely, J.F., Doschek, G.A., Gursky, H.: 1992, The potential for plasma diagnostics from stellar extreme-ultraviolet observations. Astrophys. J. Suppl. 81, 387. DOI. ADS.

Feldman, U., Doschek, G.A., Mariska, J.T., Brown, C.M.: 1995, Relationships between temperature and emission measure in solar flares determined from highly ionized iron spectra and from broadband X-ray detectors. Astrophys. J. 450, 441. DOI. ADS.

Garcia, H.A.: 1994, Temperature and emission measure from GOES soft X-ray measurements. Solar Phys. 154, 275. DOI. ADS.

Gburek, S., Siarkowski, M., Kepa, A., Sylwester, J., Kowalinski, M., Bakala, J., Podgorski, P., Kordylewski, Z., Plocieniak, S., Sylwester, B., Trzebinski, W., Kuzin, S.: 2011a, Soft X-ray variability over the present minimum of solar activity as observed by SphinX. Solar Syst. Res. 45, 182. DOI.

Gburek, S., Sylwester, J., Kowalinski, M., Bakala, J., Kordylewski, Z., Podgorski, P., Plocieniak, S., Siarkowski, M., Sylwester, B., Trzebinski, W., Kuzin, S.V., Pertsov, A.A., Kotov, Y.D., Farnik, F., Reale, F., Phillips, K.J.H.: 2011b, SphinX soft X-ray spectrophotometer: Science objectives, design and performance. Solar Syst. Res. 45, 189. DOI.

Gburek, S., Sylwester, J., Kowalinski, M., Bakala, J., Kordylewski, Z., Podgorski, P., Plocieniak, S., Siarkowski, M., Sylwester, B., Trzebinski, W., Kuzin, S.V., Pertsov, A.A., Kotov, Y.D., Farnik, F., Reale, F., Phillips, K.J.H.: 2013, SphinX: The solar photometer in X-rays. Solar Phys. 283, 631. DOI. ADS. 
Jakimiec, J., Sylwester, B., Sylwester, J., Serio, S., Peres, G., Reale, F.: 1992, Dynamics of flaring loops. II Flare evolution in the density-temperature diagram. Astron. Astrophys. 253, 269. ADS.

Kepa, A., Sylwester, J., Sylwester, B., Siarkowski, M., Kuznetsov, V.: 2005, DEM Distributions for short and long duration flares as determined from RESIK soft X-ray spectra. In: Proceedings of the 11th European Solar Physics Meeting. The Dynamic Sun: Challenges for Theory and Observations, 87.

Kotov, Y.D.: 2011, Scientific goals and observational capabilities of the CORONAS-PHOTON solar satellite project. Solar Syst. Res. 45, 93. DOI.

Kowalinski, M.: 2012, SphinX X-ray spectrophotometer. In: Romaniuk, R. (ed.) Photonics Applications in Astronomy, Communications, Industry, and High-Energy Physics Experiments, Proc. SPIE, 8454

Kuzin, S.V., Bogachev, S.A., Zhitnik, I.A., Pertsov, A.A., Ignatiev, A.P., Mitrofanov, A.M., Slemzin, V.A., Shestov, S.V., Sukhodrev, N.K., Bugaenko, O.I.: 2009, TESIS experiment on EUV imaging spectroscopy of the Sun. Adv. Space Res. 43, 1001. DOI. ADS.

Kuzin, S.V., Zhitnik, I.A., Shestov, S.V., Bogachev, S.A., Bugaenko, O.I., Ignat'ev, A.P., Pertsov, A.A., Ulyanov, A.S., Reva, A.A., Slemzin, V.A., Sukhodrev, N.K., Ivanov, Y.S., Goncharov, L.A., Mitrofanov, A.V., Popov, S.G., Shergina, T.A., Solov'ev, V.A., Oparin, S.N., Zykov, A.M.: 2011, The TESIS experiment on the CORONAS-PHOTON spacecraft. Solar Syst. Res. 45, 162. DOI. ADS.

Landi, E., Phillips, K.J.H.: 2006, CHIANTI - an atomic database for emission lines. VIII. Comparison with solar flare spectra from the solar maximum mission flat crystal spectrometer. Astrophys. J. Suppl. 166, 421. DOI. ADS.

Lang, K.R.: 2001, The Cambridge Encyclopedia of the Sun, 268. ADS.

Lee, T.T., Petrosian, V., McTiernan, J.M.: 1995, The Neupert effect and the chromospheric evaporation model for solar flares. Astrophys. J. 448, 915. DOI. ADS.

Lemen, J.R., Duncan, D.W., Edwards, C.G., Friedlaender, F.M., Jurcevich, B.K., Morrison, M.D., Springer, L.A., Stern, R.A., Wuelser, J.-P., Bruner, M.E., Catura, R.C.: 2004, The solar X-ray imager for GOES. In: Fineschi, S., Gummin, M.A. (eds.) Telescopes and Instrumentation for Solar Astrophysics, Proc. SPIE 5171, 65. DOI. ADS.

Markwardt, C.B.: 2009, Non-linear least-squares fitting in IDL with MPFIT. In: Astronomical Data Analysis Software and Systems XVIII, ASP Conference Series 411, Astronomical Society of the Pacific, San Francisco, 251.

Mazzotta, P., Mazzitelli, G., Colafrancesco, S., Vittorio, N.: 1998, Ionization balance for optically thin plasmas: Rate coefficients for all atoms and ions of the elements H to NI. Astron. Astrophys. Suppl. 133, 403. DOI. ADS.

Mewe, R., Schrijver, J.: 1978, Heliumlike ion line intensities. I - Stationary plasmas. II - Non-stationary plasmas. Astron. Astrophys. 65, 99. ADS.

More, J.J.: 1977, The Levenberg-Marquardt algorithm: Implementation and theory. In: Watson, G.A. (ed.) Numerical Analysis, Lecture Notes in Mathematics 630, Springer, Berlin, 105.

Narukage, N., Sakao, T., Kano, R., Hara, H., Shimojo, M., Bando, T., Urayama, F., Deluca, E., Golub, L., Weber, M., Grigis, P., Cirtain, J., Tsuneta, S.: 2011, Coronal-temperature-diagnostic capability of the Hinode/X-ray telescope based on self-consistent calibration. Solar Phys. 269, 169. DOI. ADS.

Parker, E.N.: 1988, Nanoflares and the solar X-ray corona. Astrophys. J. 330, 474. DOI. ADS.

Peres, G., Serio, S., Vaiana, G.S., Rosner, R.: 1982, Coronal closed structures. IV - Hydrodynamical stability and response to heating perturbations. Astrophys. J. 252, 791. DOI. ADS.

Pizzo, V.J., Hill, S.M., Balch, C.C., Biesecker, D.A., Bornmann, P., Hildner, E., Grubb, R.N., Chipman, E.G., Davis, J.M., Wallace, K.S., Russell, K., Cauffman, S.A., Saha, T.T., Berthiume, G.D.: 2005, The NOAA Goes-12 Solar X-Ray Imager (SXI) 2. Performance. Solar Phys. 226, 283. DOI. ADS.

Reale, F., Peres, G., Orlando, S.: 2001, The Sun as an X-ray star. III. Flares. Astrophys. J. 557, 906. DOI. ADS.

Reinard, A., Hill, S., Viereck, R., Bailey, S.: 2005, Calibration of GOES/SXI and XRS instruments. In: $A G U$ Fall Meeting Abstracts, B344. ADS.

Rosner, R., Tucker, W.H., Vaiana, G.S.: 1978, Dynamics of the quiescent solar corona. Astrophys. J. 220, 643. DOI. ADS.

Ryan, D.F., Milligan, R.O., Gallagher, P.T., Dennis, B.R., Tolbert, A.K., Schwartz, R.A., Young, C.A.: 2012, The thermal properties of solar flares over three solar cycles using GOES X-ray observations. Astrophys. J. Suppl. 202, 11. DOI.

Ryan, D.F., Dominique, M., Seaton, D., Stegen, K., White, A.: 2016, Effects of flare definitions on the statistics of derived flare distributions. Astron. Astrophys. 592, A133. DOI. ADS.

Sterling, A.C., Doschek, G.A., Pike, C.D.: 1994, Fe XXV temperatures in flares from the YOHKOH Bragg crystal spectrometer. Astrophys. J. 435, 898. DOI. ADS.

Sylwester, B., Sylwester, J., Siarkowski, M., Engell, A.J., Kuzin, S.V.: 2011, Physical characteristics of AR 11024 plasma based on SPHINX and XRT data. Cent. Eur. Astrophys. Bull. 35, 171. 
Sylwester, J., Kuzin, S., Kotov, Y., Farnik, F., Reale, F.: 2008, SphinX: A fast solar photometer in X-rays. Astrophys. J. Lett. 29, 339. DOI.

Sylwester, J., Kowalinski, M., Gburek, S., Siarkowski, M., Kuzin, S., Farnik, F., Reale, F., Phillips, K.J.H., Bakała, J., Gryciuk, M., Podgorski, P., Sylwester, B.: 2012, SphinX measurements of the 2009 solar minimum X-ray emission. Astrophys. J. 751, 111. DOI.

Thomas, R.J., Crannell, C.J., Starr, R.: 1985, Expressions to determine temperatures and emission measures for solar X-ray events from GOES measurements. Solar Phys. 95, 323. DOI. ADS.

van Beek, H.F., de Feiter, L.D., de Jager, C.: 1974, Hard X-ray observations of elementary flare bursts, and their interpretation. In: Rycroft, M.J., Reasenberg, R.D. (eds.) Space Research XIV, 447. ADS.

Veronig, A., Temmer, M., Hanslmeier, A., Otruba, W., Messerotti, M.: 2002, Temporal aspects and frequency distributions of solar soft X-ray flares. Astron. Astrophys. 382, 1070. DOI. ADS.

White, S.M., Thomas, R.J., Schwartz, R.A.: 2005, Updated expressions for determining temperatures and emission measures from goes soft X-ray measurements. Solar Phys. 227, 231. DOI. ADS. 